\title{
WANDERING DOMAINS IN THE ITERATION \\ OF ENTIRE FUNCTIONS
}

\section{N. BAKER}

[Received 7 February 1984]

\section{Introduction}

If $f$ is a rational function of degree at least 2 or, alternatively, a non-linear entire function, denote by $f^{n}$, for $n \in \mathbb{N}$, the $n$th iterate of $f$ regarded as a mapping of $\mathbb{C}$ to $\mathbb{C}$. Set

$$
\begin{aligned}
N(f) & =\left\{z:\left(f^{n}\right) \text { normal in some neighbourhood of } z\right\}, \\
J(f) & =\mathbb{C}-N(f) .
\end{aligned}
$$

$N(f)$ is the 'set of normality' and $J(f)$ is the 'Fatou-Julia' set for $f$. By definition, $N(f)$ is open (possibly empty). It is easily shown (see, for example, $[6,7]$ ) that $J(f)$ is non-empty and perfect, and, further, that $J(f)$ is completely invariant under mapping by $f$, by which is meant that $z \in J(f)$ implies both $f(z) \in J(f)$ and $c \in J(f)$ for any $c$ which satisfies $f(c)=z$. Also $N(f)$ is completely invariant.

If $U$ is a component of $N(f)$ then $f(U)$ lies in some component $V$ of $N(f)$ and $f(U)=V$ except in the case when $f$ is transcendental entire with a Picard-exceptional (omitted) value $c$ such that $c \in V$, when we have $f(U)=V-\{c\}$. Suppose that $f^{n+k}(U) \cap f^{n}(U) \neq \varnothing$ for some integers such that $n \geqslant 0, k>0$, and that $n, k$ are minimal with this property. Then $W=f^{n}(U)$ is periodic with period $k$ and the limiting behaviour of the iterates $\left(f^{m}\right)$ in $W$, and hence in $U$, is well understood: only a small number of different possible cases arise. These are listed, for example, in [12].

If all $f^{n}(U)$, with $n \in \mathbb{N}$, are different components of $N(f)$, the component $U$ is called a 'wandering domain' of $f$. Sullivan $[12,13]$ solved a problem open since the papers of Fatou and Julia by proving:

THEOREM A. A rational function whose degree is at least 2 has no wandering domains.

Transcendental entire functions may, however, have wandering domains. Two rather different types have been constructed.

EXAMPLE 1. This is constructed in [3] as a product

$$
g(z)=C z^{2} \prod_{n=1}^{\infty}\left(1+\frac{z}{a_{n}}\right)
$$

where the $a_{n}$ satisfy $1<a_{1}<a_{2}<\ldots$ and grow so rapidly that $a_{n+1}<g\left(a_{n}\right)<2 a_{n+1}$. The $a_{n}$ are chosen so that the annuli

$$
A_{n}: a_{n}^{2}<|x|<a_{n+1}^{\frac{1}{2}}
$$

have the property $g\left(A_{n}\right) \subset A_{n+1}$. The component $U_{n}$ of $N(g)$ which contains $A_{n}$ is a wandering domain for which $g\left(U_{n}\right)=U_{n+1}$ and $g^{k}\left(U_{n}\right) \rightarrow \infty(k \rightarrow \infty)$ hold. 
In $\S 3$ we show that any $f$ such that $N(f)$ has a multiply-connected component provides wandering domains similar to those of Example 1.

Example 2. This is due to M. Herman, and is quoted in [12]. It is $f(z)=z+2 \pi i-1+e^{-z}$. I must thank M. Herman for explaining the idea behind the construction of Example 2 and showing me its usefulness in many other situations.

Whereas, in Example 1, $g$ has very slow growth, the example of Herman has order 1. In $\S 5$ we show that for any $\rho$, with $1 \leqslant \rho \leqslant \infty$, there is an entire function $f$ of order $\rho$ which has an infinity of different families of wandering domains; that is, there are components $U_{i}$, with $i \in \mathbb{N}$, of $N(f)$, such that $f^{m}\left(U_{i}\right)=f^{n}\left(U_{j}\right)$, for $m, n \in \mathbb{N}$, implies that $i=j$ and $m=n$. All the components in this example and in Herman's are simplyconnected.

In discussing these examples one comes upon an interesting problem about commuting functions, that is, functions $f, g$ such that $f(g)=g(f)$. If $f$ and $g$ are rational, it is easy to see that $J(f)=J(g)$, but the proof does not obviously extend to the case when $f$ and $g$ are transcendental entire. In $\S 4$ we show that $J(f)=J(g)$ does indeed hold in a very special but useful case when $f, g$ are entire.

It seems natural to seek to extend Sullivan's proof of Theorem A to cover some classes of entire functions. In $\S 6$, we analyse the proof to extract a set of conditions on the entire function $f$ under which Sullivan's method shows that $f$ has no wandering domains. It is shown that these conditions are satisfied if $f$ has the form

$$
f(z)=\int^{z} P(t) \exp (Q(t)) d t,
$$

where $P$ and $Q$ are polynomials. The form $f=P_{1} e^{Q_{1}}$ for polynomials $P_{1}, Q_{1}$ is a special case of this. By a further argument one can show that $P\left(e^{z}\right)$ has no wandering domains if $P$ is a polynomial.

\section{Results from iteration theory}

Where not otherwise stated these results are proved in $[6,7]$.

Definition. A number $c \in \widehat{\mathbb{C}}$ is Fatou-exceptional for $f$ if $f^{n}(z)=c$ has only a finite set of solutions for all values $n \in \mathbb{N}$ taken together. If $f^{\prime}$ is rational, there are at most two such values, and if $f$ is transcendental entire, there is at most one finite $c$ with this property.

LeMma 2.1. Given any $z \in J(f)$ and any $w$ which is not Fatou-exceptional for $f$, there is a sequence $z_{n_{k}}$, with $n_{k} \in \mathbb{N}$, such that

$$
f^{n_{k}}\left(z_{n_{k}}\right)=w, \quad z_{n_{k}} \rightarrow z \quad\left(n_{k} \rightarrow \infty\right) .
$$

Lemma 2.2. If $\alpha \in J(f)$, if $N$ is an open neighbourhood of $\alpha$, and if $K$ is a compact plane set which does not contain a Fatou-exceptional point of $f$, then there exists $n_{0}$ such that $f^{n}(N) \supset K$ for all $n$ such that $n>n_{0}$.

Definition. The number $z_{1}$ is a fixed point of order $p$ of $f$ if $f^{p}\left(z_{1}\right)=z_{1}$ and $f^{k}\left(z_{1}\right) \neq z_{1}$ for $k<p$. If $z_{1} \neq \infty$, the value of $\left(f^{p}\right)^{\prime}\left(z_{1}\right)$ is called the multiplier of $z_{1}$. If $\left|\left(f^{p}\right)^{\prime}\left(z_{1}\right)\right|>1$ holds, $z_{1}$ is called repulsive. 
Lemma 2.3. (See [2] for entire functions.) The repulsive fixed points form a dense subset of $J(f)$.

LEMMA 2.4. If $z_{1}$ is a fixed point of order 1 of $f$ and if $\left|f^{\prime}\left(z_{1}\right)\right|<1$ then $z_{1}$ belongs to a component $U$ of $N(f)$ such that

(i) $f^{n}(z) \rightarrow z_{1}$ in $U$, and

(ii) $U$ contains a singular point of $f^{-1}$.

If we replace $\left|f^{\prime}\left(z_{1}\right)\right|<1$ by $f^{\prime}(z)=1$, the statements remain true, except that $z_{1}$ is on the boundary of $U$ and not in the interior.

\section{Entire functions with multiply-connected domains of normality}

THEOREM 3.1. If $f$ is transcendental entire and $U$ is a multiply-connected component of $N(f)$, then $U$ is a wandering domain. Further, $f^{n} \rightarrow \infty$ in $U(n \rightarrow \infty)$ and, for large $n, f^{n}(U)$ contains a closed curve $\gamma_{n}$ whose distance from 0 is large and whose winding number about 0 is non-zero. In this case every component of $N(f)$ is bounded.

COROLlaRY. If the entire function $f$ has a path to $\infty$ on which $f$ is bounded, then all components of $N(f)$ are simply-connected.

One may note that the situation in Theorem 1 is similar to that in the special Example 1 quoted in the introduction.

Proof of the theorem. It follows from the maximum principle that $U$ can be multiply-connected only if $f^{n} \rightarrow \infty$ in $U$. In [4] it was shown further that if $U$ is multiply-connected, then every component of $N(f)$ is bounded. Thus $U$ must be a wandering domain of $f$.

Denote by $\gamma$ a Jordan curve in $U$ whose interior contains points of $J(f)$. By Lemma 2.3 there is a fixed point $\alpha$ of some order $p$ inside $\gamma$. For every $n, f^{n}(\alpha)$ takes one of the values $\alpha_{i}=f^{i}(\alpha)$, where $i=1,2, \ldots, p$. For large $n$ the curve $f^{n}(\gamma)$ has large distance from 0 , since $f^{n} \rightarrow \infty$ on $\gamma$, and by the argument principle $f^{n}(\gamma)$ has non-zero winding number about $f^{n}(\alpha)$. Thus $f^{n}(\gamma)$ winds about 0 if $n$ is large.

\section{Fatou-Julia sets for commuting functions}

If $f, g$ are rational and $f(g)=g(f)$ then $J(f)=J(g)$ [8]. It is an open problem whether the same is true when 'rational' is replaced by 'entire'.

Suppose as usual that $f, g$ are rational or entire, not linear or bilinear. Suppose also throughout the section that $f(g)=g(f)$.

LeMma 4.1. $g(J(f)) \subset J(f)$.

For, by Lemma 2.3, $\alpha \in J(f)$ if and only if $\alpha$ is a limit point of repulsive fixed points of $f$. But $g$ maps a repulsive fixed point $\beta$ of order $p$ of $f$ to another fixed point $\beta^{\prime}$ such that $\left(f^{p}\right)^{\prime}(\beta)=\left(f^{p}\right)^{\prime}\left(\beta^{\prime}\right)$.

Lemma 4.2. If $f, g$ are rational then $g(N(f)) \subset N(f)$.

Proof. If $\alpha \in N(f)$, let $D$ be a disc of centre $\alpha$, radius $\rho>0$, such that $D \subset N(f)$. Then $g(D)$ is a neighbourhood of $g(\alpha)$. Since $f^{n}$ is normal in $N(f)$ we can find for any 
$\delta>0$ a sufficiently small $\rho$ such that the (spherical) diameter of $f^{n}(D)$ is at most $\delta$, for $n=1,2, \ldots$. Since $g$ is uniformly continuous in the spherical metric we may, for a given $\varepsilon>0$, ensure that $\delta>0$ above is such that the diameter of $g\left(f^{n}(D)\right)=f^{n}(g(D))$ is at most $\varepsilon$. Thus $f^{n}$ is normal in $g(D)$ which includes $g(\alpha)$.

(Obviously we cannot use the uniform continuity of $g$ on the sphere in the transcendental case.)

Remark. If $f, g$ are rational, Lemmas 4.1 and 4.2 imply that $g(J(f))=J(f)$ and $g(N(f))=N(f)$, and hence that $g^{-1}(J(f))=J(f)$. By Lemma 2.1 any $\beta \in J(g)$ is a limit point of a sequence $\left\{g^{-n}(\xi)\right\}$ for some $\xi$ which we may choose in $J(f)$. By the above, all $g^{-n}(\xi) \in J(f)$ and so $\beta$ belongs to the closed set $J(f)$. Thus $J(g) \subset J(f)$ and the symmetry of the assumptions gives $J(g)=J(f)$.

An obvious modification of Lemma 4.2 gives

LEMMA 4.3. If $f$ and $g$ are transcendental entire, then

(i) if $\alpha \in N(f)$ and there is a subsequence $f^{n_{k}}$, with $n_{k} \rightarrow \infty$, which has a finite limit in the component of $N(f)$ which contains $\alpha$, then $g(\alpha) \in N(f)$,

(ii) if $\infty$ is never a limit function of a subsequence of $\left(f^{n}\right)$ in a component of $N(f)$, then $g(N(f)) \subset N(f)$.

Proof. (i) Take $\alpha \in N(f)$ and an open neighbourhood $U$ of $\alpha$ such that $\bar{U} \subset N(f)$; then since $f^{n_{k}}$ has a finite limit function in $U$, all $f^{n_{k}}(U)$ lie in a single compact set on which $g$ is uniformly continuous. As in Lemma 4.2 one may take $U$ so small that $g\left(f^{n_{k}}(U)\right)=f^{n_{k}}(g(U))$ has small diameter for all large $n_{k}$. Lemma 2.2 then shows that $g(U)$ and, in particular, $g(\alpha)$ belong to $N(f)$.

Part (ii) follows immediately.

LEMMA 4.4. If $f$ and $g$ are transcendental entire and if $\infty$ is not a limit function of any subsequence of $\left(f^{n}\right)$ in a component of $N(f)$, nor of a subsequence of $\left(g^{n}\right)$ in a component of $N(g)$, then $J(f)=J(g)$.

This follows from Lemmas 4.1 and 4.3 by the argument given in the Remark above.

We can deal with infinite limits in one very special case which will be useful later.

Lemma 4.5. Suppose that $f$ and $g$ are entire, $f$ commutes with $g$, and $f=g+c$, where $c$ is some constant. Then $J(f)=J(g)$.

Proof. It is enough to show $g(N(f)) \subset N(f)$. Take $\alpha \in N(f)$, and a neighbourhood $U$ of $\alpha$ such that $\bar{U} \subset N(f)$. By Lemma 4.3 (i) we need consider only the case when $f^{n} \rightarrow \infty$ in $U$. Take a constant $A$ such that $A>|c|+1$. There exists $n_{0}$ such that $\left|f^{n}\right|>A$ holds in $U$ for $n>n_{0}$, and hence $|f(z)|>A$ holds for $z \in f^{n}(U)$, with $n>n_{0}$.

If $g(\alpha) \notin N(f)$, then $\left(f^{m}\right)$ takes all values, with at most one exception, for arbitrarily large $m$ in $g(U)$. Thus there exists $t=g(\xi)$, with $\xi \in U$, such that, for some $m>n_{0}$,

$$
\left|f^{m}(t)\right|=\left|f^{m}(g(\xi))\right|=\left|g\left(f^{m}(\xi)\right)\right|<1 .
$$

Thus $\eta=f^{m}(\xi) \in f^{m}(U)$ and $|g(\eta)|<1$. But $|f(\eta)|>A$ and so

$$
|c|=|f(\eta)-g(\eta)|>A-1,
$$


which contradicts the choice of $A$. The conclusion is that $\alpha \in N(f)$ implies $g(\alpha) \in N(f)$.

\section{Examples of Herman's type}

These examples may be derived from the commutative diagram

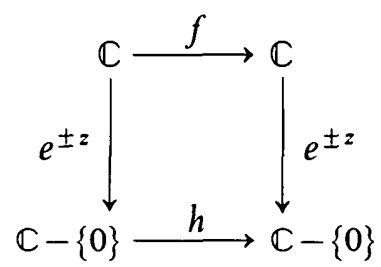

in which $\mathbb{C}$ can be regarded as a covering space of $\mathbb{C}-\{0\}$ and $f$ is an analytic map compatible with the covering property, that is an entire function of the type $f(z)=z+P(z)$, where $P$ is entire with period $2 \pi i$. The map $h$ is then entire in many cases and one may transfer a description of the iterative behaviour of $h^{n}$ to that of $f^{n}$, or vice versa, although some care has to be exercised. If $h$ is not entire then it has the form

$$
h(t)=t^{n} \exp \left\{F(t)+G\left(t^{-1}\right)\right\}
$$

where $F, G$ are entire, and the iterates $h^{n}$ are defined and map $\mathbb{C}-\{0\}$ into itself. This, apart from entire or rational maps, is the only case in which one can set up a nontrivial iteration theory of the Fatou-Julia type, based on the theory of normal families, as was pointed out by Rådström [11]. Thus the method may also be used to provide examples of Rådström's case by carrying over known results from the entire case.

EXAMPLE 5.1 (Example 2 of $\S 1$ ). Taking $f(z)=z-1+e^{-z}$ in the diagram and $e^{-z}$ as the projection map gives $h(t)=e^{-t} e^{-t}$, which has a superattractive fixed point at $t=1$. The domain of attraction of $t=1$ is easily seen to contain the positive real axis. In the iteration of $f$ this fixed point lifts to an infinity of superattractive fixed points $z_{n}=2 n \pi i$, for $n \in \mathbb{Z}$. Each $z_{n}$ has as its domain of attraction a different component $D_{n}$ of $N(f)$. Clearly $N(f)$ is translated into itself by $z \rightarrow z+2 \pi i$ since

$$
f^{n}(z+2 \pi i)=f^{n}(z)+2 \pi i \text {. }
$$

Thus $D_{n+1}=D_{n}+2 \pi i$ and $f^{n}(z) \rightarrow z_{k}$ for $z \in D_{k}$. (In fact all these statements about $f$ are clear without any reference to the way in which $f$ was constructed from $h$.)

Put $g(z)=2 \pi i+f(z)$ so that $f(g)=g(f)$ and, by Lemma $4.5, J(f)=J(g)$ and $N(f)=N(g)$. Thus $D_{n}$ is a component of $N(g)$ and $g\left(D_{n}\right)=2 \pi i+f\left(D_{n}\right)=D_{n+1}$, so that each $D_{n}$ is a wandering domain for $g$. Note that each $D_{k}$ is simply-connected, in fact approximately a 'horizontal strip' which goes to $\infty$ in both directions.

THEOREM 5.2. For any $\rho$ such that $1 \leqslant \rho \leqslant \infty$ there exists an entire function $f$ of order $\rho$, which has an infinity of different families of wandering domains: there exist components $U_{i}$, where $i \in \mathbb{N}$, of $N(f)$, such that $f^{m}\left(U_{i}\right)=f^{n}\left(U_{j}\right)$, with $i, j, m, n \in \mathbb{N}$, implies that $m=n$ and $i=j$.

Proof. Take $f_{j}(z)=2 \pi j i+z+\left(K\left(e^{z}\right)\right)^{2}$ where $j$ is an integer and $K$ is entire. Taking $e^{z}$ for the projection in the commuting diagram gives $h(t)=t \exp \left(K^{2}(t)\right)$. Now $f_{0}$ and 
$f_{1}$ commute and so, by Lemma 4.5, $J\left(f_{0}\right)=J\left(f_{1}\right)$. Also $J\left(f_{0}\right)$ is invariant under translation by $2 \pi i$ since $f_{0}^{n}(z+2 \pi i)=f_{0}^{n}(z)+2 \pi i$. We take $K$ so that $K(t)=0$ has an infinity of solutions $t=\alpha_{1}, \alpha_{2}, \ldots$. Then $h$ has fixed points of order 1 , multiplier $h^{\prime}\left(\alpha_{n}\right)=1$ at each $\alpha_{n}$, and these lift to families of fixed points $z_{n, k}=\beta_{n}+2 \pi i k$, where $\beta_{n}$ is a fixed determination of $\log \alpha_{n}$. It is also easy to check directly that for each $z$ of this form one has $f_{0}(z)=z$ and $f_{0}^{\prime}(z)=1$.

By Lemma 2.4, $z_{n, k}$ is on the boundary of at least one component $U_{n, k}$ of $N\left(f_{0}\right)$, such that $f_{0}$ maps $U_{n, k}$ to itself and $f_{0}^{n} \rightarrow z_{n, k}(n \rightarrow \infty)$ in $U_{n, k}$. Thus $U_{n, j}=U_{m, i} \neq \varnothing$ if and only if $m=n$ and $i=j$. We may choose the components $U_{n, k}$ so that $U_{n, k}+2 \pi i=U_{n, k+1}$.

Now take $f=f_{1}$ and we have $f\left(U_{n, k}\right) \subset U_{n, k+1}$, so that $U_{n, k}$ is a wandering domain for $f$. The components $U_{i, 0}$ have the property claimed in the theorem.

It only remains to show that we can prescribe the order of $K\left(e^{z}\right)$, which is the same as that of $f$, to have the required value.

Suppose that $1<\rho<\infty$. Set

$$
K(z)=\prod_{j=1}^{\infty}\left(1+\frac{z}{a_{j}}\right),
$$

where $a_{j}$ is an increasing positive sequence such that the counting function $n(r)$ of the number of $a_{j}$ in $\{z:|z| \leqslant r\}$ satisfies

$$
n(r) \sim \rho(\log r)^{\rho-1}, \quad \text { as } r \rightarrow \infty .
$$

The averaged counting function $N(r)$ is given by

$$
N(r)=\int_{0}^{r} \frac{n(t)}{t} d t \sim(\log r)^{\rho}, \quad \text { as } r \rightarrow \infty .
$$

Now (see, for example, [5, p. 47]) the maximum modulus function

satisfies

$$
M(r)=\max _{|z|=r}|K(z)|=K(r)
$$

where

$$
N(r) \leqslant \log M(r) \leqslant N(r)+Q(r),
$$

$$
\begin{aligned}
Q(r) & =r \int_{r}^{\infty} t^{-2} n(t) d t \\
& <2 r \int_{r}^{\infty} \rho t^{-2}(\log t)^{\rho-1} d t \\
& =2 r\left[\frac{\rho}{r}(\log r)^{\rho-1}+\int_{r}^{\infty} \frac{\rho(\rho-1)}{t^{2}}(\log t)^{\rho-2} d t\right]
\end{aligned}
$$

on integration by parts. Thus, as $r \rightarrow \infty$,

$$
Q(r)(1-o(1))<2 \rho(\log r)^{\rho-1}=o(N(r)),
$$

and

$$
\log M(r) \sim N(r) \sim(\log r)^{\rho}, \quad \text { as } r \rightarrow \infty .
$$

The maximum modulus function for $K\left(e^{z}\right)$ is given by $M_{1}(r)=K\left(e^{r}\right)$, so that

$$
\log M_{1}(r) \sim \log M\left(e^{r}\right) \sim r^{\rho}, \quad \text { as } r \rightarrow \infty .
$$


This completes the proof of Theorem 5.2 in the case where $1<\rho<\infty$. If $\rho=1$, we choose the $a_{j}$ so that $n(r)$ grows more slowly than any power of $\log r$, for example, so that $n(r) \sim \log \log r$, which leads to $\log M_{1}(r) \sim r \log r$. On the other hand, $n(r) \sim(\log r)^{\log \log r}$ leads to $\rho=\infty$.

EXAMPLE 5.3. If in the commuting diagram we take $f_{n}(z)=z+2 n \pi+\lambda \sin z$ and if we take $e^{i z}$ for the projection map, then $h(t)=t \exp \left\{\frac{1}{2} \lambda\left(t-t^{-1}\right)\right\}$. Here $h$ is a mapping of Rådström's type, and has a fixed point $t=1$ with multiplier $h^{\prime}(1)=1+\lambda$. For $f_{0}$ there are corresponding fixed points above $t=1$, that is at $z=2 n \pi$, where $n \in \mathbb{Z}$, with multiplier $(1+\lambda)$. If we take $|1+\lambda|<1$, the fixed points at $2 n \pi$ are attractive and each belongs to a component $U_{n}$ of $J\left(f_{0}\right)$ in which $f_{0}^{m} \rightarrow 2 n \pi(m \rightarrow \infty)$, and $f_{0}\left(U_{n}\right) \subset U_{n}$.

Further, we have $f_{0}^{n}(z+2 \pi)=f_{0}^{n}(z)+2 \pi$, so that $J\left(f_{0}\right)$ is invariant under translation by $2 \pi$. Once again $f_{1}$ and $f_{0}$ commute so that $J\left(f_{1}\right)=J\left(f_{0}\right)$. Thus $f_{1}$ maps $U_{n}$ into $U_{n+1}$, which is therefore a wandering domain for $f_{1}$.

\section{Some classes of entire functions without wandering domains}

An analysis of Sullivan's proof of Theorem A allows an application to at least some entire cases.

THEOREM 6.1. Suppose that the transcendental entire function $f$ has the following properties:

(i) the singularities of $f^{-1}$ lie over finitely many points;

(ii) the components of $N(f)$ are simply-connected;

(iii) there are a constant $\lambda>1$ and a finite set of real parameters $X_{1}, \ldots, X_{k}$ such that every entire function of the form $f_{\Phi}=\Phi f \Phi^{-1}$, where $\Phi$ is $K$-quasiconformal, for $1 \leqslant K \leqslant \lambda$, and $\Phi$ fixes $0,1, \infty$, can be expressed uniquely in terms of $X_{1}, \ldots, X_{k}$;

(iv) if in (iii) $\Phi=\Phi(t, z)$ depends on a system of parameters $t$ which vary in an open neighbourhood $T$ of the origin of $\mathbb{R}^{M}$ for some $M$, in such a way that $\Phi(0, z)=z$ and $\Phi(t, z) \in C^{1}(T)$ for fixed $z$, then in some open subset of $T$ the parameters $X_{i}$ are also $C^{1}$-functions of $t$.

Then $f$ has no wandering domains.

We postpone the proof until the next section.

Theorem 6.2. If $P$ and $Q$ are polynomials, with $Q$ non-constant, then

$$
f(z)=\int_{0}^{z} P(t) e^{Q(t)} d t
$$

satisfies the assumptions of Theorem 6.1 and thus has no wandering domains.

Proof. (i) The singularities of $f^{-1}$ are of two types. The algebraic singularities are of the form $f(c)$ where $f^{\prime}(c)=0$, and are clearly finite in number. The transcendental singularities correspond to the different asymptotic paths of $f$. Since $f$ has finite order, the number of transcendental singularities is also finite by the theorem of Denjoy, Carleman and Ahlfors.

(ii) There are paths on which $f(z)$ is bounded as $z$ approaches $\infty$ and so by the corollary to Theorem 3.1 every component of $N(f)$ is simply-connected. 
(iii) We may write $P(z)=\prod\left(z-z_{i}\right)^{m_{i}}$ and $\sum m_{i}=p=\operatorname{deg} P$. Let $\Phi$ be a normalized $K$-quasiconformal mapping such that $g=f_{\Phi}=\Phi f \Phi^{-1}$ is entire. Then $g$ is a local homeomorphism except at $\Phi\left(z_{i}\right)$ and in the neighbourhood of such points $g$ is an $m_{i^{-}}$ to-1 map. Thus the only zeros of $g^{\prime}$ are those of the polynomial

$$
\tilde{P}(z)=\prod_{i}\left(z-\Phi\left(z_{i}\right)\right)^{m_{i}},
$$

of degree $p$.

Now (see, for example, [10]) there is a constant $C$ such that, for large $z$, $|\Phi(z)|<C|z|^{K}$ and $\left|\Phi^{-1}(z)\right|<C|z|^{K}$. If the degree of $Q$ is $q$, so that $f$ has order $q$, this implies that the order of $g$ is at most $K q$. Since $g^{\prime}$ has only finitely many zeros, the order of $g^{\prime}$ (which equals the order of $g$ ) is an integer and, provided that $K q<q+1$, this must be $q$. Thus if $K<(q+1) / q$, we have

$$
g^{\prime}(z)=\tilde{P}(z) \exp (\tilde{Q}(z)), \quad \operatorname{deg} \tilde{Q}=q .
$$

Since $f(0)=0$ and $\Phi(0)=0$, it follows that $g(0)=0$, and so

$$
g(z)=\int_{0}^{z} \tilde{P}(u) e^{\tilde{Q}(u)} d u,
$$

which is characterized by $(p+q+2)$ complex parameters, the coefficients of $\tilde{P}$ and $\tilde{Q}$.

(iv) Suppose now that $\Phi_{t}(z)=\Phi(t, z)$ depends continuously differentiably on parameters $t \in T \subset \mathbb{R}^{M}$ as in the statement of the theorem. Then the representation for $\tilde{P}$ obtained in (iii) shows that the coefficients $\mathbf{b}$ of $\tilde{P}$ are in $C^{1}(T)$.

Since $f^{\prime}$ has only a finite number of zeros, it is easily shown that $f(z)=z$ has an infinity of solutions (see, for example, [9, Theorem 3.2]). Note that, by Lemma 2.4, only finitely many of these fixed points have $f^{\prime}(z)=1$. If $\alpha_{i}$ are the various fixed points of $f$ then $\beta_{i}(t)=\Phi\left(t, \alpha_{i}\right)$ are those of $\Phi_{t} f \Phi_{t}^{-1}$. If the coefficients of $\tilde{Q}$ are $\mathbf{a}=\left(a_{0}, a_{1}, \ldots, a_{q}\right)$ then each $\beta_{i}$ gives rise to an equation

$$
F_{i}(\mathbf{b}, \mathbf{a}, t) \equiv \int_{0}^{\beta_{i}(t)} \tilde{P}(u) \exp (\tilde{Q}(u)) d u=\beta_{i}(t) .
$$

We shall prove that there exists a choice of $(q+1)$ of the $\alpha_{i}$ so that, at $t=0$,

$$
D=\operatorname{det}\left(\frac{\partial F_{i}}{\partial a_{j}}\right)_{0 \leqslant i, j \leqslant q} \neq 0 .
$$

This is sufficient to prove that the coefficients a of $\widetilde{Q}$ are in $C^{1}(T)$ bear 0 .

If $D=0$ at $t=0$, no matter how we choose $(q+1)$ of the $\alpha_{i}$, then there is a maximum number $m<q+1$ of linearly independent rows $\left\{\partial F_{i} / \partial a_{j}: 0 \leqslant j \leqslant q\right\}$. There is a non-zero vector $\left(v_{0}, \ldots, v_{q}\right)$ orthogonal to these rows, and hence to all other such rows for the infinity of possible choices of $i$. Thus

$$
G(z)=\int_{0}^{z} \sum_{j=0}^{q} v_{j} u^{j} P(u) \exp (Q(u)) d u
$$

is zero whenever $f(z)=z$. We have used the fact that $\tilde{P}$ and $\tilde{Q}$ reduce to $P$ and $Q$ when $t=0$.

Since $f(z)-z=0$ has at most finitely many multiple roots, we have

$$
\frac{G(z)}{f(z)-z}=\frac{E(z)}{H(z)}
$$


where $E$ is entire, of order at most $q$, and $H$ is a polynomial, whose degree we denote by $h$.

Write $\gamma=\arg a_{q}(0)$. For values of $z=r e^{i \theta}$ in a sector where $\cos (q \theta+\gamma)>\delta>0$ one has, on integrating by parts,

$$
\int_{0}^{z} u^{j} \exp (Q(u)) d u \sim z^{j}(\exp Q(z)) / Q^{\prime}(z), \quad \text { as } z \rightarrow \infty,
$$

and so $E(z) \sim$ Const. $z^{h+j^{\prime}}$, where $j^{\prime}$ is the greatest value of $j$ such that $v_{j} \neq 0$. In a sector where $\cos (q \theta+\gamma)<-\delta$ one has $E(z)=O(1) z^{h-1}$. Phragmén-Lindelöf arguments show that for a function $E$ of order $q$ these growth relations imply that $E$ is a polynomial. But a polynomial cannot have different growth in the different types of sector, as above. Thus in fact $D$ cannot be zero at $t=0$.

THEOREM 6.3. If $p$ is a non-constant polynomial, then $f(z)=p\left(e^{2}\right)$ has no wandering domains.

Proof. Suppose that this is not the case and that $U_{1}$ is a component of $N(f)$ such that all $U_{n}=f^{n-1}\left(U_{1}\right)$, for $n \geqslant 1$, are different. Clearly $N(f)$ is invariant under translation by $2 \pi i$.

Suppose that for some integers $j, k, l$,

$$
\varnothing \neq U_{j} \cap\left(U_{k}+2 \pi i l\right) .
$$

Then $U_{j+1}=f\left(U_{j}\right)$ meets $U_{k+1}=f\left(U_{k}+2 \pi i l\right)$, which is possible only if $j=k$. Thus $\exp \left(U_{j}\right)$ and $\exp \left(U_{k}\right)$ are disjoint if $j \neq k$.

We have the commuting diagram

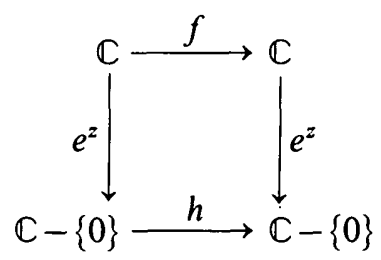

where $h(t)=\exp (p(t))$. Hence $h$ maps $V_{n}=\exp U_{n}$ to $V_{n+1}=\exp U_{n+1}$. In $V_{k},\left(h^{n}\right)$ takes no values which lie in $V_{k}$, for $n>1$. Thus $\left(h^{n}\right)$ is normal in each $V_{k}$.

In fact each $V_{k}$ is a component of $N(h)$. For any boundary point of $V_{k}$ has the form $e^{\alpha}$, where $\alpha$ is a boundary point of $U_{k}$. But $\alpha$ is a limit point of fixed points $z_{n}$ of $f: f^{v_{n}}\left(z_{n}\right)=z_{n}$. Since $\exp \left(f^{v}\right)=h^{v}(\exp )$ we have $\exp \left(z_{n}\right)=h^{v_{n}}\left(\exp z_{n}\right)$, and $\exp \left(z_{n}\right) \rightarrow e^{\alpha}$, which is thus a limit point of fixed points of $h$ (of arbitrarily large orders) and so belongs to $J(h)$.

Thus the $V_{k}$ are wandering components of $h$; but by Theorem 6.2 no such components exist. The theorem is proved.

\section{Proof of Theorem 6.1}

The proof depends on constructing a large family of quasiconformal maps each of which conjugates the given function to another entire function. We recall some definitions and results about quasiconformal maps. See, for example, $[10]$.

Let $\kappa$ denote a number such that $0 \leqslant \kappa<1$ and write $K=(\kappa+1) /(1-\kappa)$. A topological map $\varphi$ of a domain $D$ into $C$ is $K$-quasiconformal if and only if $\varphi$ is 
absolutely continuous on horiztonal and vertical lines and if $\left|\varphi_{\bar{z}}\right| \leqslant\left|\varphi_{z}\right|$ a.e. in $D$, where $\varphi_{\bar{z}}=\frac{1}{2}\left(\varphi_{x}-i \varphi_{y}\right)$ and $\varphi_{z}=\frac{1}{2}\left(\varphi_{x}+i \varphi_{y}\right)$. The quantity $\mu=\varphi_{\bar{z}} / \varphi_{z}$, which exists almost everywhere in $D$, is the 'complex dilatation' of $\varphi$. The property that $\varphi$ is conformal is equivalent to the vanishing of $\mu$ a.e.

LEMMA 7.1. Given quasiconformal maps $\varphi: G \rightarrow H$ and $\psi: H \rightarrow K$ we have that $\psi \varphi$ is quasiconformal and

(i) if $\psi$ is conformal then $\mu_{\psi \varphi}=\mu_{\varphi}$ a.e.,

(ii) if $\varphi$ is conformal then $\mu_{\psi \varphi}=\mu_{\psi}(\varphi) \overline{\varphi^{\prime}(z)} / \varphi^{\prime}(z)$ a.e.,

(iii) if $\varphi, \psi$ are surjective, then if $\mu_{\psi \varphi}=\mu_{\varphi}$ a.e., we have $\psi$ conformal.

This implies

LEMMA 7.2. If $f$ is a one-to-one conformal map of $D$ onto $D_{1}$, if $\varphi$ is quasiconformal and defined on $D \cup D_{1}$, and if $\mu=\mu_{\varphi}$ satisfies

$$
\mu(f)=\mu(z) f^{\prime}(z) / \overline{f^{\prime}(z)} \text { a.e. in } D,
$$

then $\varphi f \varphi^{-1}$ is conformal in $\varphi(D)$.

Lemma 7.3 (The measurable Riemann mapping theorem). Given a measurable function $\mu$ on the plane such that $\|\mu\|_{\infty}<1$, there exists a unique sense-preserving quasiconformal homeomorphism $\varphi=\varphi^{\mu}$ of $\hat{\mathbb{C}}$ to. $\widehat{\mathbb{C}}$ such that $\varphi_{\bar{z}}=\mu \varphi_{z}$ and $\varphi$ fixes $0,1, \infty$.

Ahlfors and Bers [1] extended this to allow for a dependence on parameters. Their result includes the following lemma.

LEMMA 7.4. Write $t=\left(t_{1}, \ldots, t_{n}\right)$ and $s=\left(s_{1}, \ldots, s_{n}\right)$. Suppose that for all $t$ in some open set $T$ of $\mathbb{R}^{n}$ there is a function $\mu(t, z)$, with $z \in \mathbb{C}$, measurable with respect to $z$, such that $\|\mu(t, z)\|_{\infty} \leqslant 1$, and that (suppressing $z$ )

$$
\mu(t+s)=\mu(t)+\sum_{i=1}^{n} a_{i}(t) s_{i}+|s| \alpha(t, s)
$$

with $\|\alpha(t, s)\|_{\infty} \leqslant c$ for some constant $c$, and $\alpha(t, s) \rightarrow 0$ a.e. in $z$ as $s \rightarrow 0$. Suppose also that $\left\|a_{i}(t+s)\right\|_{\infty}$ are bounded and that $a_{i}(t+s) \rightarrow a_{i}(t)$ a.e. for $s \rightarrow 0$.

Then $\varphi^{\mu(t)}$ is in $C^{1}(T)$ as a function of $t$ for fixed $z$.

\section{Proof of Theorem 6.1}

Suppose that $f$ satisfies the hypotheses of Theorem 6.1 and that there is a wandering component $U$ of $N(f)$. By assumption all components of $N(f)$ are simplyconnected. Since $f^{-1}$ has only finitely many singular points it follows that, for large $n$, $f^{n}(U)$ is free of such singularities and the map $f: f^{n}(U) \rightarrow f^{n+1}(U)$ is a homeomorphism. Without loss of generality we may suppose that this is the case for $n \geqslant 0$.

Define the equivalence relation $\sim$ on $\mathbb{C}$ by $x \sim y$ if and only if there exist $m, n$ in $\mathbb{N}$ such that $f^{m}(x)=f^{n}(y)$. A class of equivalence [x] meets $U$ in at most one point. For $x, y$ in $U$ with $x \sim y$ implies that $f^{m}(x)=f^{n}(y)$ for some positive integers $m, n$. By the definition of wandering domain $m=n$, while $f^{m}$ is a homeomorphism and so $x=y$. 
Lemma 7.5. Given measurable $\mu$ in the above-mentioned $U$, such that $|\mu|<\kappa<1$ in $U$, there is an extension of $\mu$ to a function in $L^{\infty}(\mathbb{C})$, which satisfies

$$
\mu(f(z))=\mu(z) f^{\prime}(z) / \overline{f^{\prime}(z)} \text {, a.e. in } \mathbb{C} \text {. }
$$

Further $\|\mu\|_{\infty}=\sup _{U}|\mu| \leqslant \kappa$.

For define $\mu(z)=0$ if $[z] \cap U=\varnothing$ or if $[z]$ is one of the finite set of classes which contains a singular point of $f^{-1}$. For any other class $[z] \cap U=\{x\}$ for some $x \in U$ and there are positive $m, n$ such that $f^{m}(x)=f^{n}(z)$.

Define

$$
\mu(x)\left(f^{m}\right)^{\prime}(x) / \overline{\left(f^{m}\right)^{\prime}(x)}=\mu(z)\left(f^{n}\right)^{\prime}(z) / \overline{\left(f^{n}\right)^{\prime}(z)} .
$$

This extension of $\mu$ is measurable in each $f^{ \pm n}(U)$ and so in $\mathbb{C}$ and satisfies the requirements of the lemma.

LEMMA 7.6. The quasiconformal homeomorphism $\varphi^{\mu}$ associated as in Lemma 7.3 with the extended $\mu$ of Lemma 7.4 is such that

$$
f_{\mu}=\varphi^{\mu} f\left(\varphi^{\mu}\right)^{-1}
$$

is entire.

By Lemma 7.2, $f_{\mu}$ is conformal at all points except those of the form $\varphi^{\mu}(\alpha)$ where $f^{\prime}(\alpha)=0$. Thus $f_{\mu}$ is analytic except for a finite number of points in whose neighbourhood $f_{\mu}$ is bounded. Thus $f_{\mu}$ is in fact entire.

Initial construction of quasiconformal maps in the unit disc $D$

Take an integer $M>k$, where $k$ is the number of parameters in the statement of Theorem 6.1 (iii). Set

$$
T=\left\{\left(t_{1}, \ldots, t_{M}\right): t_{i} \in R,\left|t_{i}\right|<1,1 \leqslant i \leqslant M\right\} .
$$

Take three points lying on $\partial D$ in positive cyclic order, say $a, b, c$. On the arc $(a, b)$ pick arg $a<\theta_{1}<\theta_{2}<\ldots<\theta_{2 M}<\arg b$. Denote $\left[\theta_{2 j-1}, \theta_{2 j}\right]$ by $I_{j}$ and take a real $C^{1}$-function $\delta_{j}(\theta)$, where $1 \leqslant j \leqslant M$, such that, for a preassigned positive $\varepsilon, \delta_{j}=0$ except in $I_{j}, \delta_{j}>0$ in $i_{j}, \delta_{j}(\theta)+\theta<\theta_{2 j}, \theta-\delta_{j}(\theta)>\theta_{2 j-1}$ in $\check{I}_{j}$, and $\left|\delta_{j}^{\prime}(\theta)\right|<\varepsilon / 2 M<1$. Note that, for $t \in T, \theta+\sum_{1}^{M} t_{j} \delta_{j}(\theta)$ is a monotone map of $[0,2 \pi]$ to itself.

Define the homeomorphism of $D$ onto $D$ given by

$$
\varphi\left(t, r e^{i \theta}\right)=r \exp i\left\{\theta+\sum_{i}^{M} t_{j} \delta_{j}(\theta)\right\},
$$

and note that different $t$ give different $\varphi$, all of which fix $a, b, c$. Also $t \neq 0$ gives $\varphi \neq$ Id.

Each $\varphi$ is quasiconformal in $D$, for $\varphi \in C^{1}(D)$, except at $z=0$, and

$$
\begin{aligned}
\mu_{\varphi}=\varphi_{\bar{z}} / \varphi_{z} & =e^{2 i \theta}\left\{\varphi_{r}+\frac{i}{r} \varphi_{\theta}\right\} /\left\{\varphi_{r}-\frac{i}{r} \varphi_{\theta}\right\} \\
& =-\left\{e^{2 i \theta} \sum_{1}^{M} t_{j} \delta_{j}^{\prime}(\theta)\right\} /\left\{2+\sum_{1}^{M} t_{j} \delta_{j}^{\prime}(\theta)\right\} .
\end{aligned}
$$

Thus in $D,\left|\mu_{\varphi}\right| \leqslant \frac{1}{3} \varepsilon$. We choose $\varepsilon$ so that, for any $K$-quasiconformal map $\Phi$ of the plane with $K<(1+\varepsilon) /(1-\varepsilon)$ the function $f_{\Phi}$ of Theorem 6.1 (iii) depends on the $k$ 
parameters $X_{1}, \ldots, X_{k}$. We also choose $\varepsilon$ so small that all $K \leqslant\{(1+\varepsilon) /(1-\varepsilon)\}^{2}$ satisfy Lemma 7.9 (to be proved later).

\section{The plane maps}

First carry $\varphi$ over to the wandering domain $U$ as follows. From now on $\psi$ denotes a fixed one-to-one conformal map of $D$ onto $U$. Write $\varphi_{t}(z)=\varphi\left(t, r e^{i \theta}\right)$ so that, by Lemma 7.1,

$$
\chi_{t}=\psi \varphi_{t} \psi^{-1}: U \rightarrow U
$$

is quasiconformal with dilatation

$$
\mu_{t}=\mu_{\varphi_{t}}\left(\psi^{-1}\right) \overline{\left(\psi^{-1}\right)^{\prime}} /\left(\psi^{-1}\right)^{\prime},
$$

which is measurable in $U$ and satisfies $\left|\mu_{t}\right| \leqslant \frac{1}{3} \varepsilon$.

By Lemma 7.5 we extend $\mu_{t}$ to the plane (keeping the same notation) so that $\left\|\mu_{t}\right\|_{\infty}<\frac{1}{3} \varepsilon$ and, by Lemma 7.3, obtain the associated plane map $\Phi_{t}=\varphi^{\mu_{t}}$, which fixes $0,1, \infty$ and makes $f_{t}=\Phi_{t} f \Phi_{t}^{-1}$ entire, fixed by prescribing the parameters $X_{1}, \ldots, X_{k}$.

Direct computation shows that $\mu_{t}$ satisfies the requirements of Lemma 7.4. In fact either $\mu_{t}(z)=0$ for all $t$, or there exists an $x$ in $U$ such that, for some $m, n \in \mathbb{N}$,

$$
\begin{aligned}
\mu_{t}(z) & =\left(f^{m}\right)^{\prime}(x) \overline{\left(f^{n}\right)^{\prime}(z)} \mu_{t}(x) /\left(f^{n}\right)^{\prime}(z) \overline{\left(f^{m}\right)^{\prime}(x)} \\
& =e^{i \gamma(z)}\left(\sum_{1}^{M} t_{j} \beta_{j}\right) /\left(2+\sum_{1}^{M} t_{j} \beta_{j}\right),
\end{aligned}
$$

where $\gamma(z)$ is real and $\beta_{j}=\delta_{j}^{\prime}\left(\arg \psi^{-1}(x)\right)$ is constant in $t$ and $\left|\beta_{j}\right|<\varepsilon / 2 M$.

We have proved the following result.

Lemma 7.7. (i) $\Phi_{t}(z)$ is in $C^{1}(T)$ for each fixed $z$.

(ii) $\Phi_{0}(z) \equiv z$.

(iii) $f_{t}(z)=\Phi_{t} f \Phi_{t}^{-1}=F\left(X_{1}, \ldots, X_{k}, z\right)$, where, in some open subset $T^{\prime}$ of $T$, the parameters $X_{i}$ are in $C^{1}(T)$.

The third part follows from our assumptions of Theorem 6.1 (iii),(iv).

COROLLARY. Since $M>k$ there is some non-constant arc $t=t(\sigma)$, with $0 \leqslant \sigma<\sigma_{0}$, in $T^{\prime} \subset T$ on which $X(t)$ is constant.

From the corollary we have, for $0 \leqslant \sigma<\sigma_{0}$,

$$
\Phi_{t(\sigma)} f \Phi_{t(\sigma)}^{-1}=f_{t(\sigma)}=f_{t(0)}=\Phi_{t(0)} f \Phi_{t(0)}^{-1},
$$

and hence

$$
\Omega_{\sigma} f=f \Omega_{\sigma},
$$

where $\Omega_{\sigma}=\Phi_{t(0)}^{-1} \Phi_{t(\sigma)}$

Thus, for $p \in \mathbb{N}, \Omega_{\sigma} f^{p}=f^{p} \Omega_{\sigma}$. If $f^{p}(\alpha)=\alpha$, this implies that $f^{p}\left(\Omega_{\sigma}(\alpha)\right)=\Omega_{\sigma}(\alpha)$. Since the fixed points of $f^{p}$ (for given $p$ ) form a discrete set and since $\Omega_{\sigma}$ is continuous in $\sigma$, it follows that $\Omega_{\sigma}(\alpha)=\Omega_{0}(\alpha)=\alpha$. Thus $\Omega_{\sigma}$ fixes every fixed point of every order of $f$ and consequently all points of $J(f)$. Similarly $\Omega_{\sigma}$ maps $U$ into itself (and fixes every point of $\partial U$ ).

Lemma 7.8. The map $G=\psi^{-1} \Omega_{\sigma} \psi: D \rightarrow D$, with $0 \leqslant \sigma<\sigma_{0}$, fixes each point of $\partial D$. 
The proof of Lemma 7.8 follows an idea of Peter Jones, which depends on

LEMMA 7.9. There is $a K>1$ such that for every $K$-quasiconformal map $f$ of the plane which fixes $0,1, \infty$ one has $|f(z)-z|<\frac{1}{2}$ uniformly for $z \in L=\left\{e^{i \theta}: \frac{1}{3} \pi \leqslant \theta \leqslant \frac{5}{3} \pi\right\}$.

For the $K$-quasiconformal maps of given $K$ which fix $0,1, \infty$ form a normal family. If $K \rightarrow 1$ such maps must converge to $z$ uniformly on $L$.

Proof of Lemma 7.8. Each $\Phi_{t}^{ \pm 1}$ has

$$
K<(1+\varepsilon) /(1-\varepsilon)
$$

and $\Omega_{\sigma}$ is thus $\{(1+\varepsilon) /(1-\varepsilon)\}^{2}$-quasiconformal and satisfies the hypotheses of Lemma 7.9 .

Consider $z$ (close to $\partial D$ in $D$ ) and $G(z)$. Denoting the hyperbolic distance by $[\cdot, \cdot]$ we have

$$
[z, G(z)]_{D}=[\psi(z), \psi(G(z))]_{U}=\left[\psi(z), \Omega_{\sigma} \psi(z)\right]_{U} .
$$

Denote by $\alpha$ a nearest point of $\partial U$ to $\psi(z)$ and set $r=|\alpha-\psi(z)|$. There is a point $\beta$ on $\partial U$ whose distance from $\alpha$ is $r$. Scaling Lemma 7.10 gives that, since $\Omega_{\sigma}$ fixes $\alpha, \beta, \infty$, we have

$$
\left|\Omega_{\sigma}(\psi(z))-\psi(z)\right|<\frac{1}{2} r .
$$

Now $\left[\psi(z), \Omega_{\sigma}(\psi(z))\right]_{U} \leqslant\left[\psi(z), \Omega_{\sigma}(\psi(z))\right]_{w}$, where $W$ is the disc $|w-\psi(z)|<r$, and hence less than an absolute constant, independent of $z$. This implies that as $z \rightarrow \zeta \in \partial D$ we have $G(z) \rightarrow \zeta$ also.

\section{Conclusion of the proof}

For all $\sigma$ such that $0 \leqslant \sigma<\sigma_{0}, \Phi_{t(\sigma)}$ maps $U$ to the same domain $V$. Let $\hat{\psi}$ be a conformal map of $D$ onto $V$. Then Lemma 7.8 shows that $\psi^{-1} \Phi_{t(0)}^{-1} \hat{\psi} \hat{\psi} \hat{\psi}^{-1} \Phi_{t(\sigma)} \psi$ is the identity on $\partial D$. Thus

$$
\hat{\psi}^{-1} \Phi_{t(\sigma)} \psi=\hat{\psi}^{-1} \Phi_{t(0)} \psi
$$

holds on $\partial D$ and both sides are maps of $D$ to $D$ with dilatations $\mu_{t(\sigma)}$ and $\mu_{t(0)}$, respectively, which are the same as the dilatations of $\varphi_{t(\sigma)}$ and $\varphi_{t(0)}$.

By Lemma 7.1 (iii) we have

$$
\begin{aligned}
& \hat{\psi}^{-1} \Phi_{t(\sigma)} \psi=L_{\sigma} \varphi_{t(\sigma)}, \\
& \hat{\psi}^{-1} \Phi_{t(0)} \psi=L_{0} \varphi_{t(0)},
\end{aligned}
$$

where $L_{\sigma}, L_{0}$ are Moebius transformations which map $D$ to $D$. Thus on $\partial D$ we have $L_{\sigma} \varphi_{t(\sigma)}=L_{0} \varphi_{t(0)}$. But on the $\operatorname{arc}(b, c)$ of $\partial D$ both $\varphi_{t(\sigma)}$ and $\varphi_{t(0)}$ are the identity and so $L_{\sigma}$ and $L_{0}$ must be the same transformation. But this implies that $\varphi_{t(\sigma)}=\varphi_{t(0)}$ holds on the whole of $\partial D$ for $0 \leqslant \sigma<\sigma_{0}$, which contradicts the construction of the $\varphi$. The proof is now complete.

\section{References}

1. L. AHLFORS and L. Bers, 'Riemann's mapping theorem for variable metrics', Ann. of Math., 72 (1960), 385-404.

2. I. N. BAKER, 'Repulsive fixpoints of entire functions', Math. Z., 104 (1968), 252-256. 
3. I. N. BAKER, 'An entire function which has wandering domains', J. Austral. Math. Soc. Ser. A, 22 (1976), 173-176.

4. I. N. BAKER, 'The domains of normality of an entire function', Ann. Acad. Sci. Fenn. Ser. A I Math., 1 (1975), 277-283.

5. R. P. BoAs, Entire functions (Academic Press, New York, 1954).

6. P. Fatou, 'Sur les équations fonctionelles', Bull. Soc. Math. France, 47 (1919), 161-271; 48 (1920), 33-94, 208-314.

7. P. Fatou, 'Sur l'itération des fonctions transcendantes entières', Acta Math., 47 (1926), 337-370.

8. G. Julia, 'Mémoire sur la permutabilité des fractions rationelles', Ann. Sci. École Norm. Sup. (3), 39 (1922), 131-215.

9. W. K. Hayman, Meromorphic functions (The Clarendon Press, Oxford, 1964).

10. O. Lehto and K. I. VIRTANEN, Quasikonforme Abbildungen (Springer, Berlin, 1965).

11. H. RÅDSTRÖM, 'On the iteration of analytic functions', Math. Scand., 1 (1953), 85-92.

12. D. Sullivan, 'Itération des fonctions analytiques complexes', C. R. Acad. Sci. Paris Sér. A-B, 294 (1982), 301-303.

13. D. SULlivan, 'Quasiconformal homeomorphisms and dynamics I. Solution of the Fatou-Julia problem on wandering domains', preprint M/82/59, Institut des Hautes Études Scientifiques, Paris, November 1982.

\author{
Department of Mathematics \\ Imperial College of Science and Technology \\ Huxley Building \\ Queen's Gate \\ London SW7 2BZ
}

